\title{
Incidencias de la perspectiva de género en la educ ación legal. Un recorrido exploratorio sobre el campo jurídico en Argentina ${ }^{(*)}$
}

\author{
Incidents of the gender perspective in legal education. An exploratory tour \\ of the legal field in Argentina
}

\author{
Malena Costa Wegsman ${ }^{(*)}$ \\ Argentina - Universidad de Buenos Aires
}

\begin{abstract}
Resumen: La década de 1990 marca un punto de inflexión en cuanto a la incidencia de la categoría de género en el discurso jurídico. En América Latina en general, y en Argentina en particular, comienza a producirse desde entonces, de manera sostenida, toda una serie de sanciones a nivel legislativo, que tienen, desde ya, antecedentes diversos. La promulgación de esas legislaciones se entrelaza virtuosamente con otros planos de la vida política, como las reglamentaciones que se dan en las instituciones públicas y el funcionariado, los reclamos y las distintas acciones llevadas a cabo por todo el amplio arco de movimientos sociales, partidos políticos y sindicatos. La universidad y la formación académica también juegan un papel clave en la transformación del campo jurídico en relación con la igualdad de género y las disidencias sociosexuales. Hacia finales del siglo $X X$, en algunos programas de estudio de varias universidades latinoamericanas, se vislumbra de manera explícita cierto vocabulario vinculado con los estudios de género y de las sexualidades. En Argentina, la articulación entre el género y el derecho comienza a perfilarse como un campo de conocimientos a partir del retorno a la democracia en 1983, aunque se consolida durante la década de 1990 y, con mayor contundencia, en estos últimos años, en virtud de una variedad de acontecimientos relativos al posicionamiento de los feminismos en la vida política.
\end{abstract}

En este texto se presenta un análisis exploratorio de la incursión de los contenidos de género y de los estudios de las sexualidades y feministas en la academia jurídica argentina. El propósito general es ofrecer un recorrido en torno a los avances de la denominada perspectiva de género en la formación universitaria, considerando las complejidades políticas y conceptuales que tal perspectiva conlleva.

Palabras clave: Educación - Feminismos - Género - Derecho - Argentina

Abstract: The 1990s marked a turning point in terms of the impact of the gender category in legal discourse. In Latin America in general, and in Argentina overall, a series of sanctions at the legislative level began to take place since then, all of which have a diverse background. The enactment of these laws is intertwined with other stages of political life, such as the regulations implemented in public institutions and civil services, as well as the claims and many actions carried out throughout a wide range of social movements, political parties and work unions. University and academic education also

$\left({ }^{*}\right) \quad$ Nota del Editor: este artículo fue recibido el 10 de octubre de 2019 y su publicación fue aprobada el 30 de octubre de 2019.

$\left(^{* *}\right.$ Profesora y Doctora en Filosofía por la Universidad de Buenos Aires (Argentina), con mención en Estudios de Género. Investigadora posdoctoral de la Universidad de Buenos Aires. Miembro del Instituto Interdisciplinario de Estudios de Género. Contacto: malenacostaw@gmail.com 
played a key role in the transformation of the legal field in relation to gender equality and socio-sexual dissidence. Towards the end of the twentieth century, in some study programs of several Latin American universities, the vocabulary linked to gender studies and studies of sexualities is envisioned. In Argentina, the linkage between gender and law begins to emerge as a field of knowledge since the return to democracy in 1983, although it is consolidated during the 1990s and, with greater force, in recent years, based on a variety of events related to the positioning of feminisms in political life.

This text submits an exploratory analysis of the incursion of gender and feminist content in the Argentine legal academy. The general purpose is to offer a review on the advances of the so-called gender perspective in university instruction, the political and conceptual complexities that such perspective entails.

Keywords: Education - Feminisms - Gender - Law - Argentina

\section{Introduc ción}

El propósito general de este texto es ofrecer un recorrido en torno a los avances de la denominada perspectiva de género en la formación universitaria, considerando las complejidades políticas y conceptuales que tal perspectiva conlleva. Para ello, se presenta un análisis exploratorio de la incursión de los contenidos de género y de los estudios de las sexualidades y feministas en la academia jurídica argentina. Los tres presupuestos que marcan el punto de partida de este análisis se constituyen a partir de algunos hallazgos de la investigación posdoctoral en curso sobre los feminismos jurídicos en Argentina (Costa, 2017; Costa, 2019; Costa \& Lerussi, 2018).

En primer lugar, respecto de la progresión del derecho en relación con las sexualidades, la década de 1990 marca un punto de inflexión en cuanto a la incidencia de la categoría de género en el discurso jurídico. En América Latina en general, y en Argentina en particular, comienza a producirse desde entonces, y de manera sostenida, toda una serie de sanciones a nivel legislativo, que tienen, desde ya, antecedentes diversos. En segundo lugar, se observa que la promulgación de esas legislaciones se entrelaza virtuosamente con otros planos de la vida política, como las reglamentaciones que se dan en las instituciones públicas y el funcionariado, los reclamos y las distintas acciones llevadas a cabo por todo el amplio arco de movimientos sociales, partidos políticos y sindicatos. Tercero, considerando el eje de la investigación en curso, se pone de relieve que la universidad y la formación académica juegan un papel clave en la transformación del campo jurídico en relación con la igualdad de género y las disidencias sociosexuales. En efecto, hacia finales del siglo $X X$, en algunos programas de estudio de varias universidades latinoamericanas, se vislumbra de manera explícita cierto vocabulario vinculado con los estudios de género y de las sexualidades. En Argentina, la articulación entre el género y el derecho comienza a perfilarse como un campo de conocimientos a partir del retorno a la democracia en 1983, aunque se consolida durante la década de 1990 y, con mayor contundencia, en estos últimos años, en virtud de una variedad de acontecimientos relativos al posicionamiento de los feminismos en la vida política.

Todas esas transformaciones exhiben la compleja interrelación de los diversos escenarios que conforman el campo jurídico: los distintos estratos del poder estatal, el campo del activismo entrelazado por las acciones de organizaciones sociales, los colectivos militantes a causa de la movilización legal en términos amplios y, por fin, las comunidades académicas en las que se pugna por preservar, modificar e instituir determinadas líneas de pensamiento y modalidades de enseñanza-aprendizaje del conocimiento jurídico. Para considerar los modos en que se asume la perspectiva de género en Argentina es necesario atender a los efectos que a partir de ese compromiso se producen en cada una de esas instancias. Así, en este texto, se pone de relieve en el primer apartado, la contundencia del género sobre el campo jurídico a partir de la vinculación general del derecho con una cultura androcéntrica predominante. En el segundo apartado, se analizan las incursiones del pensamiento feminista en el conocimiento jurídico de la academia estadounidense como un antecedente ineludible de los estudios de género en el campo. Luego, en el tercer y cuarto punto, se describen los avances del género en las universidades de América Latina y de Argentina, respectivamente, dando cuenta de las transformaciones que esta categoría supone sobre el arco político, considerando tanto las promulgaciones legislativas y las políticas públicas, así como los escenarios políticos y jurídicos en que estas se llevan a cabo. En el quinto apartado, se indaga sobre las posibilidades de la perspectiva de género en la educación legal en las universidades 
argentinas para, finalmente, señalar los efectos paradojales que la asunción de dicho enfoque supone desde un posicionamiento feminista crítico.

\section{Lo que encubre la neutralidad jurídica}

El derecho se construye como una trama discursiva que regula la vida de los ciudadanos, individuos libres e iguales en tanto sujetos racionales y, en tal sentido, el derecho se convierte en una pieza fundamental del mundo moderno. En función de resguardar la imparcialidad legal, el discurso jurídico ostenta la neutralidad como un principio impostergable. No obstante, esa pretensión de neutralidad ha sido blanco de críticas desde la consumación del Estado moderno. Un ejemplo paradigmático de las más tempranas críticas jurídicas lo brinda Olympe de Gouges con su Declaración de los derechos de la Mujer y la Ciudadana de 1791, donde pone de relieve la omisión de las mujeres encubierta tras la presunta neutralidad del derecho. En efecto, la regulación legal implica la negación de toda una serie de grupos, desplazados a una posición subalterna en la medida en que no gozan de las características definidas para el ideal del ciudadano. Desde el pensamiento feminista, se evidencia que el sujeto normativo de la Modernidad, presentado en su carácter abstracto y universal, se corresponde con la condición de varón, blanco, adulto, burgués, capaz y, preferentemente, heterosexual y subscripto a la religión predominante.

Esta operatoria de subalternación jurídica se mantiene vigente hasta nuestros días, oscilando entre lo explícito y lo tácito, lo evidente y lo subrepticio. A partir de las consideraciones de los estudios de género y feministas, se hace posible advertir el carácter intrínsecamente androcéntrico del derecho en función de su sostenida complicidad para perdurar los privilegios del sujeto normativo. Así, se revela que, tanto el establecimiento del varón como universal de lo humano, así como la negación de toda otra identificación sociosexual en las formulaciones jurídicas, son efecto del androcentrismo del derecho. El androcentrismo jurídico se evidencia en la regulación restringida de las normas y procedimientos legales, en la parcialidad que predomina en el funcionamiento de las instituciones de la justicia y en los sesgos que demarcan los cánones del derecho. La composición preponderante de los ámbitos oficiales del conocimiento del derecho, masculino, blanco y burgués, también es una señal del androcentrismo jurídico (Costa, 2015).

El acceso tardío de grupos diferentes del varón normativo a las instituciones del conocimiento jurídico puede comprenderse como efecto de la equiparación de esta disciplina con lo masculino. Así lo advierte Francis Olsen, quien señala que, alejado de su postulada neutralidad, El sexo del derecho (2000) se corresponde con el del sujeto normativo de la Modernidad. El binarismo sexual que define una humanidad en la oposición macho-hembra se colma a través de ciertas características prescriptas para cada una de esas posibilidades excluyentes. Así, los atributos de la racionalidad, la actitud activa, la capacidad de pensamiento abstracto, el poder y la objetividad se corresponden con los atributos del varón-macho, cifrados en las condiciones de universalidad propias del sujeto moderno. Contrariamente, Io irracional o una racionalidad deficiente, el predominio de la emoción, la incapacidad o dificultad para el pensamiento abstracto y una marcada sensibilidad son todos rasgos que se asocian con lo femenino, y se asimilan a lo particular, situado a la fuerza de la naturaleza, dadivosa y nutricia, pero peligrosa, en su indocilidad. Puesto que el derecho es erigido como una disciplina científica, la neutralidad, objetividad y universalidad, los cuales son requisitos del dominio de la ciencia y rasgos atribuidos a la masculinidad, se convierten en una condición inexcusable de este campo. Por consiguiente, las identificaciones sociosexuales femeninas y feminizadas quedan limitadas en su participación de lo jurídico. La transformación de esta lógica de subalternación es, precisamente, el leitmotiv de las intervenciones feministas en el derecho.

\section{Intervenciones feministas en el campo jurídico}

\subsection{Los debates por la inclusión y el riesgo esencialista}

El concepto de esencialismo es elaborado desde el pensamiento feminista para aludir a aquellas posturas que sostienen que lo que iguala y articula al amplio conjunto de las mujeres es una esencia fundamental. El mayor riesgo respecto del esencialismo es, en resumidas cuentas, la homogenización de todas las formas en que se puede vivir la experiencia del ser mujer. En efecto, una postura esencialista, por definición, anula las diversidades en función de sostener una concepción unívoca de mujer o de cualquier identificación sociosexual. En tal sentido, resulta problemático que la argumentación a favor del acceso de grupos excluidos a las instituciones modernas se corresponda con una búsqueda de consecuencias específicas. 
Incidencias de la perspectiva de género en la educación legal. Un recorrido exploratorio sobre el campo jurídico en Argentina

Incidents of the gender perspective in legal education. An exploratory tour of the legal field in Argentina

El razonamiento para la inclusión de sectores tradicionalmente relegados, basado en las ventajas esenciales que estos podrían producir, es endeble desde el punto de vista político, en tanto que supone asunciones esencialistas; pero también es estratégico y empírico, por cuanto que no es corroborable desde cualquier enfoque crítico (Kohen, 2000). Por consiguiente, desde una posición feminista crítica, la urgencia de garantizar el acceso de los grupos marginados a la institucionalidad jurídica se sostiene no en base a ciertos beneficios específicos que esa inclusión podría acarrear, sino, en todo caso, a favor de la igualdad y con el objetivo de corroer y desmantelar los privilegios androcéntricos.

\subsection{Los feminismos jurídicos en la academia legal}

Con todo, aun tomando en cuenta la advertencia antiesencialista, es asimismo innegable que el acceso y la inclusión de aquellos grupos marginados genera una rotunda transformación en los espacios donde habían sido negados previamente. Esa transformación se concreta, en primer lugar, en una embestida contra los criterios de exclusión vigentes; y en segundo lugar, en la restitución de igualdad a través de la apertura a las posiciones y críticas que esos grupos puedan ofrecer. Los efectos del acceso masivo de mujeres a las universidades durante la segunda mitad del siglo $X X$ constituyen un claro ejemplo de esa transformación. En efecto, a partir de la década de 1960, la entrada de mujeres a la academia del norte occidental transforma las tradiciones del conocimiento instituido, al promover la circulación de novedosas ideas feministas, que influencian todos los campos del saber. Más puntualmente, en las universidades de Estados Unidos, la incidencia de los feminismos obtiene un temprano reconocimiento a través de la inauguración de un dominio académico especializado. Hacia el final de la década de 1970, en algunas universidades de ese país, se instituye un área de producción académica denominada Feminist Jurisprudence, Feminist Legal Theory o Feminist Legal Thought (FLT) (Bartlett \& Kennedy et al., 1991), a la que en castellano se llama Feminismos Jurídicos (Costa, 2016).

Los aportes de los feminismos jurídicos al campo del derecho se articulan a través de una serie de debates en torno a casos judiciales, reformas legislativas y movilización legal. A través de un acervo heterogéneo de publicaciones, encuentros académicos e intervenciones varias, se da cause y visibilidad a las discusiones feministas sobre el derecho, permeando no sólo los contenidos académicos sino, además, distintos alegatos judiciales. Así, por ejemplo, las intervenciones de abogadas feministas en las demandas de Geduldig vs. Aiello, 417 U.S. 484 (1974) o General Electric Company v. Gilbert 429 U.S. 125 (1976), sobre discriminación sexual en el ámbito laboral, ponen en discusión las nociones de igualdad resguardadas por la Constitución estadounidense, más precisamente, por la Cláusula de Igual Protección de la 14ta Enmienda, y coadyuvan de manera invaluable en la proclamación de la (controvertida) Ley de Discriminación por Embarazo (Pregnancy Discrimination Act de 1978).

\subsection{Tres principios generales sobre género y derecho}

Si bien en América Latina no se registran paralelos institucionales de los feminismos jurídicos, es decir, no se registran áreas de investigación académica específica de feminismos jurídicos; sí existen diversos espacios de formación y circulación de conocimiento definido en el cruce entre género y derecho. En esa intersección se produce una corriente de pensamiento en la que se corrobora una notable continuidad con los presupuestos epistemológicos que sustentan las propuestas jurídicas feministas estadounidenses. Estos postulados pueden cifrarse en tres posicionamientos generales. El primer postulado menciona que desde los feminismos se sostiene que las identificaciones sociosexuales distintas del sujeto normativo moderno son ignoradas durante siglos por el discurso del derecho. En tal sentido, desde las distintas posturas feministas, se advierte la falsedad en la proclamación del principio de neutralidad jurídico. El segundo postulado afirma una relación indisoluble entre teoría y práctica. Desde esta afirmación, cualquier intervención en el campo del derecho se considera como praxis, es decir, afectada tanto por las condiciones materiales como por los principios y conocimientos que pone en juego quien actúa o interviene jurídicamente. Esta tesis confronta con el objetivismo de la ciencia jurídica liberal predominante, que es caracterizada desde los feminismos no solo como androcéntrica, sino también como clasista, racista y heterocentrista. De la consideración del derecho como praxis y el imposible en su neutralidad se infiere su condición inherentemente política, esto es, indisociable de los intereses de quienes lo construyen, interpretan y aplican. En consecuencia, desde los feminismos también se asume la necesaria confluencia de diversos discursos en la construcción de la trama jurídica. La articulación deliberada de distintas disciplinas es un tercer postulado feminista en el campo jurídico. Estas ideas circulan y sustentan la articulación del género en el campo del derecho latinoamericano. 


\section{Género y derecho en América latina}

La movilización social en torno a los derechos emerge en América latina con una marcada fuerza a partir del accionar del movimiento de Derechos Humanos, en relación con los crímenes de las dictaduras militares y con la vigencia de la ley en medio de los enfrentamientos armados entre la guerrilla y las fuerzas del orden estatal (Costa, 2016). Esta movilización posibilita, entre otros aspectos, una familiarización con el lenguaje jurídico por parte de diversos sectores sociales en Latinoamérica, lo que facilita, posteriormente, planteamientos de problemáticas relativas a la igualdad de género. Hacia la década de 1980, las restituciones democráticas se consolidan a través del accionar de agrupaciones que trabajan para profundizar el Estado y la política de derechos recobrados. Un caso paradigmático es el de las Madres y las Abuelas de Plaza de Mayo en Argentina, quienes adecuaron las exigencias de respeto a los Derechos Humanos con los reclamos por el derecho a la identidad de sus hijas/os y nietas/os.

La década de 1990 en la región latinoamericana se caracteriza por el proceso de internacionalización del derecho, reforzado por los efectos de algunas de las conferencias de la Organización de Naciones Unidas (ONU). Los encuentros auspiciados por la ONU contribuyen también a la internacionalización del movimiento de mujeres, característica que se afianza, sobre todo, con las Conferencias de Viena (1993), El Cairo (1994) y, en particular, la IV Conferencia Internacional sobre la Mujer de Beijing (1995). Los lineamientos de esta última conferencia quedan plasmados en una Plataforma de Acción que propone "la integración de una perspectiva de género en las políticas relacionadas con todas las áreas de la sociedad, así como en la aplicación de medidas positivas con ayuda institucional y financiera adecuada en todos los niveles" (Informe de la IV Conferencia Mundial sobre la Mujer Beijing, cit. en Rodríguez Manzano, 2008, pág. 219-220). De esta forma, el género es concebido como la base de un enfoque que debe adoptarse en todas las políticas de gobierno, incluso en los programas de formación.

El paralelismo entre la internacionalización del derecho y el internacionalismo feminista convergen en la introducción de la categoría de género en América Latina. A mediados de la década de 1990, se incorpora el género como una categoría de uso frecuente en los textos académicos y gubernamentales de la región. En líneas generales, la categoría de género se formula desde el pensamiento feminista para dar cuenta de los modos relacionales, culturales y políticos en que se establecen las diferencias sexuales $y$, sobre todo, para dilucidar las maneras en que dichas diferencias producen desigualdades. Según las corrientes feministas, los sentidos de esta categoría se precisan una y otra vez polémicamente, los escenarios políticos e históricos también, en función de los distintos intereses de quienes la enuncian en sus discursos.
En la región latinoamericana, el género se extiende cada vez con mayor amplitud y se incorpora no solo en los discursos de las organizaciones de militancia sociopolítica $y$ en el lenguaje normativo del Estado, sino que también se vuelve cada vez más usual en los medios de comunicación e, incluso, como parte del lenguaje coloquial, fenómeno que se consolida de manera cotidiana. Esta flexibilidad categorial llevó a que en algunas oportunidades el género goce de una suerte de autonomía relativa respecto del pensamiento feminista. La difusión de la categoría de género, celebrada, por cierto, como una victoria del pensamiento para la igualdad, conlleva una consecuencia paradójica por cuanto que, en ocasiones, el género es desprendido de toda connotación política y es usado como un concepto meramente descriptivo. Esta situación se advierte en la academia jurídica argentina cuando el uso del género obedece a un cálculo estratégico por parte de las profesoras feministas para que sus ideas puedan ser recibidas. En ese sentido, muchas profesoras e investigadoras del derecho aducen la necesidad de incorporar sus propuestas en las universidades a través del mote de "estudios de género", considerado más neutral que la alusión al feminismo, a fin de lograr la aprobación de cursos por parte de las autoridades de sus casas de estudio (Costa, 2019).

Con todo, aun en su uso más desabrido, el género no deja de ser un concepto que pone de relieve las condiciones de posibilidad epistemológica de la diferencia sexual. En tal sentido, se trata de una categoría sustentada en un aspecto nodal del conflicto social, de modo que la incorporación del género al lenguaje normativo del Estado implica, aunque más no sea nominativamente, el reconocimiento de que las desigualdades emergen como efecto de concepciones opresivas de las diferencias. Por lo tanto, la incorporación del género al entramado jurídico supone, aunque sea potencialmente, una fractura en el androcentrismo del derecho. Se trata de un logro a escala internacional, sin dudas, una de las conquistas más relevantes del siglo XX para una elaboración más amplia y compleja del principio de igualdad. 


\section{Igualdad de género en Argentina}

La década de 1990 en Argentina se caracteriza por una marcada expansión política en materia de igualdad de género y por el crecimiento de la incidencia feminista en las facultades de Derecho. La influencia de los feminismos en el campo jurídico argentino se vislumbra en un marco de articulaciones políticas, en el que las investigaciones académicas se sustentan en un escenario normativo regional y local, y una trama de producción activista político-legal (Costa, 2017). En tal sentido, si bien en este país el derecho a la igualdad goza de garantía constitucional desde 1853, según lo establecen los artículos 16 y 20 de la Constitución Nacional, el reconocimiento por parte del Estado de la existencia de grupos que padecen discriminación en virtud del género se evidencia a partir de la sanción legislativa y la proclamación de derechos específicos, sobre todo desde fines de la década de 1990. Previamente, caben destacarse algunas medidas, como la ratificación de la adhesión de la Argentina a la Convención sobre la Eliminación de todas las Formas de Discriminación contra la Mujer (CEDAW) en 1985. Durante aquella década de 1980, también se celebran dos reformas del derecho civil sumamente significativas en relación con la igualdad de género, como fueron la Ley de Divorcio Vincular (Ley 23 515/87) y la de Patria Potestad Compartida (Ley 23 264/85), reconociendo la igualdad de deberes y derechos para varones y mujeres y la posibilidad para ambas partes del cuidado de sus hijas/os por fuera del matrimonio. Estas reformas son contemporáneas a otras conquistas legales para las mujeres, como el derecho a la obtención de pensiones por vínculo conyugal sin la formalización matrimonial.

La incorporación de la "perspectiva de género" en la institucionalidad estatal, según lo dispuesto en la Plataforma de Beijing, supone el reconocimiento de que el Estado, aun siendo democrático, representa de manera predominante los intereses de un sujeto normativo, concretamente encarnado en los varones y, por consiguiente, abona de manera significativa a la opresión sexual. Asumir la perspectiva de género en las políticas públicas significa que los sentidos de las sexualidades también estructuran el Estado y, en consecuencia, las desigualdades de género son parte constitutiva de sus instituciones (Rodríguez, 2008). En tal sentido, si bien en Argentina la ciudadanía política de las mujeres se consagra en 1947 mediante la obtención del derecho al sufragio, es recién hacia la década de 1990 cuando se asume que el acceso de la población femenina a las jerarquías estatales no está garantizado. A partir de esa advertencia, se aprueba en 1991 la Ley 24012 de cuotas femeninas, con la que Argentina se convierte en el primer país en el mundo en incluir a nivel nacional este tipo de normativa, que establece un porcentaje mínimo de candidatas mujeres en las listas electorales para todos los comicios. Esta medida se replica en el año 2002 en la normativa sindical, estableciendo el mismo porcentaje mínimo que la ley nacional (30\%), o bien un porcentaje proporcional al número de mujeres trabajadoras en el caso de no alcanzarse el mínimo establecido en la norma.

La reforma constitucional de 1994 también es destacable en relación con la igualdad de género, pues a partir de ella se inviste de jerarquía constitucional a una serie de instrumentos jurídicos internacionales (Artículo 75, inciso 22), entre ellos, a la CEDAW, cuyo Protocolo Facultativo será ratificado en 2006. Asimismo, dos años más tarde, la Convención Interamericana para Prevenir, Sancionar y Erradicar la Violencia contra la Mujer (Convención de Belem do Pará) se incorpora al cuerpo legislativo nacional (Ley 24 632/96).

En el Poder Judicial también se evidencia una progresiva, aunque más lenta, institucionalización del género. A partir de los primeros años del nuevo milenio, y como consecuencia de los mecanismos para garantizar el mandato de la perspectiva de género, la institucionalización del género en el Poder Judicial argentino se produce, primero, a través de una serie de políticas y oficinas con competencia en temas de mujeres y diversidad sexual, inauguradas en distintos estratos del sistema de justicia. En segundo lugar, el nombramiento en el año 2003 de dos mujeres como ministras de la Corte Suprema, por primera vez en la historia de los gobiernos democráticos en Argentina, puso de relieve la situación de desigualdad de género en la composición del funcionariado del Poder Judicial. Si bien en Argentina la representación femenina alcanza un nivel respetable en el Poder Legislativo como resultado de la implementación de la Ley de Cuotas, la situación no se reproduce en el ámbito judicial. La designación de las magistradas contribuye así a remarcar la situación de estratificación en la composición femenina de las judicaturas argentinas, a la vez que subraya la relevancia del género en la carrera judicial.

En este proceso de promulgación legislativa y jurídica en relación con el género que se despliega fuertemente durante la década de 1990 y a partir del nuevo milenio, respectivamente, resulta insoslayable la incidencia de grupos organizados a favor de los derechos que impulsan y acompañan los 
debates legislativos y los casos judiciales, en continuidad con la fuerte tradición argentina de intervención político-jurídica por parte de los movimientos sociales (Costa, 2019). A partir de estas reformas y transformaciones políticas, se vislumbra cómo la categoría de género se perfila, en un primer momento, en exclusiva relación con las mujeres. En efecto, en la confección de las políticas públicas, las denominadas Políticas para las Mujeres anteceden a las políticas de género, lo que se relaciona con el paso del enfoque de "mujeres y desarrollo" a la "perspectiva de género" en los lineamientos de los organismos internacionales (Rodríguez, 2008). Hacia fines del siglo XX, el accionar del movimiento TLGBIQ+ (trans -travesti, transexual y transgénero, según las autodenominaciones colectivas locales, lésbica, gay, bisexual, intersex y queer) posibilita la ampliación de los sentidos de la categoría de género, a fin de extenderla y referenciar con ella la subalternación que pesa sobre todas las disidencias sexuales. De modo que, si en un primer momento las nociones de discriminación y violencia de género se definen en exclusiva relación con las mujeres, durante los primeros años de este siglo XXI, estas nociones se profundizan para comprender también la discriminación y la violencia sufrida por las personas de la comunidad TLGBIQ+(1).

El accionar de estos grupos a favor de la igualdad de derechos alimenta el cúmulo de ideas en relación con el género, las sexualidades, la política y el campo jurídico. Así, en Argentina, los conocimientos producidos desde el activismo se articulan complejamente con los de los distintos ámbitos jurídicos, promoviendo el encuentro y los debates entre activistas, abogadas, juristas y distintas profesionales feministas y de la diversidad sexual, constituyendo "nuevas comunidades epistémicas" (Bergallo \& Moreno, 2017) que, a su vez, sustentan la producción jurídica del ámbito académico.

\section{Estudios de género, sexualidades y feminismos en la academia jurídica argentina}

En Argentina, la visibilidad de los feminismos, los colectivos de la disidencia sexual y las organizaciones de mujeres han crecido de manera exponencial en los últimos años. La participación masiva en los debates por las leyes de matrimonio igualitario (2010), de identidad de género (2012) y por la legalización del acceso al aborto (2018), además de la creciente adhesión a las marchas del movimiento NiUnaMenos en contra de la violencia machista y a la huelga internacional de mujeres, entre muchas otras movilizaciones, han logrado imponer cuestiones relativas a la igualdad de género, la autonomía sexual y la cultura misógina y heterosexista en un lugar destacado de la sociedad y también, desde ya, en los espacios universitarios.

Con todo, la permeabilidad de la academia jurídica parece resentirse a la hora de abrir espacio a los inconmensurables aportes que los estudios de género y los feminismos pueden producir en el campo del derecho. En efecto, la transformación de los programas de estudio se revela lenta y escasa, y las modalidades de enseñanza se mantienen firmes en la tradición, dando poco o nulo lugar a los enfoques de la educación legal feminista. Este panorama de débil renovación de lo establecido se confronta con la vibrante insistencia de profesionales del derecho, cuyos intentos por introducir contenidos de género, sexualidades y enfoques feministas en las instituciones universitarias no cesan.

En lo que sigue, se presentan algunos apuntes respecto de las posibilidades y las dificultades en la obtención de legitimidad y circulación de los estudios de género, los feminismos y los estudios de las sexualidades en la academia legal argentina(2). La relevancia de esa posibilidad radica no solo en el reconocimiento de las corrientes del pensamiento crítico más relevantes de los últimos años en el ámbito académico y político, sino también en el cumplimiento de un compromiso de Estado, por cuanto que la perspectiva de género implica un mandato que concierne a la instrucción del funcionariado jurídico y las y los operadores

(1) Esta transformación se evidencia en dos legislaciones promulgadas durante la segunda década del nuevo milenio. En primer lugar, la ley de "Matrimonio Igualitario" (26.618/10), que reconoce los derechos de todas las uniones conyugales con prescindencia del sexo de sus integrantes; y, con mayor contundencia aun, en la Ley 26.743/12, de "Identidad de género", que implica una transformación sin precedentes internacionales en el reconocimiento político de las sexualidades trans.

(2) Los aspectos generales de las incursiones de los estudios de género, de las sexualidades y feministas en la universidad legal argentina analizados en este apartado se esbozan a partir de la observación participante de eventos académicos celebrados entre los años 2014 y 2016 en universidades nacionales y de entrevistas en profundidad realizadas a actoras clave, entre los años 2016 y 2018. El criterio para la selección de las celebraciones académicas se basó en la mención de la categoría de género o similares en sus denominaciones. Las actoras entrevistadas son profesoras de derecho en universidades nacionales y manifiestan su compromiso con la perspectiva de género o se posicionan como feministas. Versiones previas de estos análisis pueden encontrarse en Costa (2017) y (2019). 
Incidencias de la perspectiva de género en la educación legal. Un recorrido exploratorio sobre el campo jurídico en Argentina

Incidents of the gender perspective in legal education. An exploratory tour of the legal field in Argentina

del Derecho, siendo la universidad uno de los espacios más significativos para esa formación.

\subsection{Marginación y descrédito. El lugar de los contenidos de género en la formación jurídica}

Los contenidos de género no ocupan un lugar preponderante en las escuelas de Derecho argentinas. Por el contrario, los programas de las carreras de grado manifiestan una cruda marginalidad de los enfoques feministas, de los estudios de las sexualidades o, más en general, de todas las temáticas relativas al género. Esa condición marginal se revela con mayor contundencia al observar la poca disponibilidad de materiales de estudio específicos. En tal sentido, un primer dato a destacar es la ausencia de revistas académicas especializadas sobre género y derecho o feminismos jurídicos dentro del amplísimo arco de revistas jurídicas. La condición de asignatura especial u optativa de estos contenidos se refleja a su vez en los dossiers o números especiales de revistas sobre género y derecho, es decir, se trata en todo caso de publicaciones eventuales. El formato que predomina dentro de esta área es el libro, principalmente, compilaciones de capítulos, sobre todo aquellos que reúnen resultados de equipos de investigación de facultades de derecho y de otros organismos estatales (como los libros publicados por el Observatorio de Género del Poder Judicial de la Ciudad de Buenos Aires, véase Bergallo \& Moreno, 2017; Maffía \& Moreno, 2019). También, desde el año 2012, la editorial jurídica Didot publica una colección de género que reúne diversos temas trabajados desde posicionamientos feministas.

La carencia de revistas especializadas y la escasez general de publicaciones son factores que condicionan fuertemente el acceso al conocimiento de estudios sobre género y Derecho y de feminismos jurídicos. En efecto, las profesionales del derecho pioneras en la introducción de estas temáticas en los programas universitarios advierten que su acercamiento a las lecturas sobre género se produjo recién en el tramo de postgrado de sus estudios, cursando otras carreras de grado además de abogacía, en eventos académicos, en charlas informales o a partir de una búsqueda personal. En resumidas cuentas, el conocimiento de los abordajes de género, sexualidades, derecho y de los feminismos jurídicos no suele producirse en la carrera jurídica de grado, sino a partir de otras instancias.

La ausencia o el lugar secundario de esos contenidos en los planes de estudio de las escuelas de derecho se relaciona con el desmerecimiento por parte de los organismos de evaluación del conocimiento universitario y de las autoridades de la academia legal. En tal sentido, se especula con cierto recelo de las jerarquías institucionales, en tanto que los estudios de género y feministas implican una crítica reflexiva radical. En efecto, se trata de propuestas epistemológicas que conllevan la revisión de todos aspectos del hacer académico y que cuestionan, asimismo, los modos tradicionales de la enseñanza legal, a la vez que interpelan la coherencia de los saberes del canon jurídico tradicional. Otra especulación en relación con la reticencia a aceptar estos contenidos en los programas universitarios advierte el descrédito hacia los feminismos en tanto una corriente de pensamiento digna de estudio, análisis y profundización. Finalmente, en la medida en que estos conocimientos no forman parte del corpus tradicional de la academia jurídica, también se sospecha sobre cierta ignorancia o desconocimiento de los estudios de género y feministas, en sentido amplio, por parte de las jerarquías universitarias.

Esa falta de contenidos de género y derecho converge con otra ausencia significativa. Las entrevistadas advierten con preocupación la insuficiente formación sobre Derechos Humanos en general en las carreras de grado. En tal sentido, no resulta sorprendente el exiguo estudio de normativas internacionales para la igualdad de género, incluso aquellas que son parte del articulado constitucional nacional, como la CEDAW.

Por fin, cabe destacar que la inclusión de estos contenidos y enfoques depende en gran medida de la voluntad de algunas y algunos profesionales del derecho, cuyo interés se concentra en que el género deje de ser una eventualidad aleatoria y se convierta, en cambio, en un enfoque transversal de la formación jurídica universitaria, tal como se supone con la asunción de la perspectiva de género. Pese a insistir con fortaleza y obstinación, su suerte se ve coartada, en primer lugar, por la falta de reconocimiento de los estudios de género, de las sexualidades y de los feminismos jurídicos como contenidos legítimos de formar parte en los programas de estudio de la academia legal. Ese desmerecimiento se manifiesta, en ocasiones, en la necesidad de definir estratégicamente los títulos de los cursos o programas propuestos. Así, nombres más generales, como perspectiva o estudios de género, logran mayor aceptación que aquellos que hacen alusión explícita a los feminismos o a las disidencias sexuales. En segundo lugar, e igualmente relevante, la falta de reconocimiento de estos contenidos se 
revela en la insuficiencia presupuestaria que predomina en la contratación de docentes expertas, la celebración de eventos específicos y la publicación de revistas especializadas.

\subsection{Posibilidades y desafíos. Debates y propuestas para una educación legal de género o feminista}

La expansión legislativa e institucional en materia de igualdad de género de los últimos años se sostuvo en paralelo con una movilización feminista amplísima y divergente. Esas transformaciones que se diseminan por los diversos planos de lo político impulsan un notable incremento en la celebración de eventos alternativos, edición de publicaciones independientes y conformación de grupos y espacios autogestivos sobre género, sexualidades y derecho y feminismos jurídicos en la academia legal argentina. Aun así, la inclusión de esos contenidos en los cánones de la disciplina legal continúa siendo dispersa y marginada.

Para abonar al reconocimiento de estos temas en la academia, es importante destacar las características singulares que el pensamiento sobre sexualidades, género y Derecho y los feminismos jurídicos presentan en este país. En tal sentido, en la bibliografía de producción local es habitual encontrar referencias a una serie de pensadoras e investigadoras de los FLT. Esas alusiones se justifican en el acuerdo con los tres postulados feministas respecto del derecho: tal como se señala más arriba, las/os profesionales del derecho de Argentina, y de América Latina en general, comprometidas/os con nociones de igualdad de género adhieren a la impugnación de la neutralidad del derecho y de la uniformidad de la trama jurídica, así como también concuerdan en la asunción del conocimiento en tanto praxis. Además de compartir esos principios generales, también se advierte la continuidad entre las pensadoras locales con temas clásicos de los feminismos y algunos enfoques de los FLT, como las críticas a la distinción jurídica entre lo público y lo privado y al sujeto del derecho (Costa \& Lerussi, 2018). Esos acuerdos y continuidades son efecto de la marcada recepción por parte de todo el campo jurídico latinoamericano de tradiciones y corrientes de pensamiento de Estados Unidos. En relación con el pensamiento feminista, más concretamente, la categoría de género es un producto de los feminismos angloparlantes del norte, traducida y recreada luego por el movimiento feminista en América Latina (Costa, 2017).

Con todo, el pensamiento jurídico de Argentina revela importantes aspectos originales, como el lugar central que encuentra la tradición de los Derechos Humanos (Costa \& Lerussi, 2018). Esa centralidad también es parte de los enfoques feministas y de género, cuya incidencia en la academia legal se sustenta en una fuerte conexión con las organizaciones activistas de la sociedad civil. En efecto, desde las iniciativas de los activismos feministas y de la disidencia sexual se genera un intercambio de ideas y cooperación de prácticas con las universidades, lo cual constituye una diferencia insoslayable respecto de las producciones del norte. Por fin, las condiciones materiales -de habitual restricción presupuestaria para estos temas, y de una economía nacional particularmente inestable- se conjugan con determinados factores geopolíticos de una territorialidad fuertemente centralizada en la capital del país, la Ciudad de Buenos Aires, conformando un escenario de producción académica singular.

La incidencia del género en la institucionalidad jurídica académica supone, asimismo, precisar los contenidos y enfoques de relevancia para la formación legal universitaria. En tal sentido, cabe apuntar algunos temas que se destacan como los más urgentes o importantes en la trama de intervenciones de género, feministas y sexodisidentes en el campo académico jurídico argentino.

Primero, como un trabajo de reconstrucción de la teoría, se advierte la importancia de rastrear las corrientes jurídicas y políticas heredadas por los feminismos locales. Esta iniciativa es parte de una tarea más amplia de elaboración de las genealogías feministas argentinas y latinoamericanas, en función de legitimar las tradiciones regionales desde posicionamientos decoloniales y visibilizar conocimientos locales diferentes de los del norte.

Segundo, otra instancia estratégica para la formación de la práctica jurídica de género y feminista lo constituyen los mecanismos de intervención en el marco normativo internacional y regional latinoamericano. Este objetivo supone, desde ya, resguardar el conocimiento de los derechos específicos dentro del articulado del derecho internacional, lo cual, como se apunta más arriba, no es el caso en las universidades argentinas. Las presentaciones a los órganos de control del derecho internacional y regional -como los "informes sombra" al Comité de la CEDAW o los informes al Mecanismo de Seguimiento de la Convención de Belém do Pará- son un ejercicio de movilización legal habitual en Argentina. Los procedimientos y contenidos de esas intervenciones forman parte del acervo jurídico de este país y, por lo tanto, su estudio supone un conocimiento más que útil en la instrucción jurídica de género y feminista. 
Incidencias de la perspectiva de género en la educación legal. Un recorrido exploratorio sobre el campo jurídico en Argentina

Incidents of the gender perspective in legal education. An exploratory tour of the legal field in Argentina

En tercer lugar, otro punto de especial atención por parte de las/os profesionales del derecho comprometidas con un enfoque de género, feminista o sexodisidente es la educación legal. Las reflexiones discurren en relación con las posibilidades de una práctica docente que alimente los posicionamientos críticos, tomando en cuenta, sobre todo, los postulados feministas sobre el Derecho. Así, se observa la férrea jerarquía que aun predomina en las casas de enseñanza del derecho, consolidada por requisitos de cumplimiento protocolar, tales como la apariencia en el vestir. Esta exigencia, que suele ser silenciosa y sobreentendida, además de abonar al elitismo habitual de los espacios universitarios, acentúa los modelos del binarismo sexogenérico ligados a la práctica de la abogacía (Costa, 2017).

Cuarto, las articulaciones y acciones conjuntas entre los activismos, la academia y la institución judicial son parte de la tradición universitaria argentina. En relación con la enseñanza de género, feminista y de las sexualidades en el derecho, se considera importante reconocer y robustecer estas redes, desde las cuales es posible reforzar los mecanismos de circulación, obtención de legitimidad y diversificación de recursos.

\subsection{Perspectivas paradojales}

En Argentina, durante los últimos años, el activismo feminista y sociosexual se ha expandido y diversificado. Tras mucho tiempo de organización sostenida, que puede rastrearse por lo menos desde comienzos del siglo $\mathrm{XX}$, el retorno a la democracia promovió nuevas posibilidades para hacer frente a las demandas por igualdad de género y, sobre todo, para proponer formas creativas de articulación. El compromiso con la perspectiva de género, las conquistas legislativas, las cada vez más variadas y masivas movilizaciones por asuntos concernientes a la igualdad en el ejercicio de derechos básicos, la autonomía corporal, la vida libre de violencia, entre otros, no solo revitalizan y consolidan las agrupaciones existentes, sino que, además, posibilitan modos originales de intervención en todos los ámbitos públicos. La extensión que adquieren los distintos sectores feministas y la disidencia sexual ocasionan, asimismo, potenciales transformaciones en la academia jurídica, no solo para la inclusión de contenidos que ya son parte de la tradición de los estudios de género, sino, además, para la elaboración de formas de enseñanza diversas, la expansión de enfoques epistemológicos y la proyección de críticas jurídicas alternativas.

Esta proliferación de ideas y posicionamientos respecto de los feminismos y de los estudios de género y de las sexualidades suponen que la inclusión de estos contenidos en los programas de formación jurídica no resulten una mera adhesión de temas y bibliografías. Por el contrario, la aceptación de algunos enfoques críticos implica asumir ciertas limitaciones en la práctica de la enseñanza universitaria que conllevaría a una transformación total del modus académico.

En efecto, ciertos aspectos inherentes a la lógica académica se revelan como contrarios o contraproducentes para el potencial transformador de ciertos posicionamientos igualitarios, feministas o de la disidencia sexual. En consecuencia, la incorporación de los estudios de género en el canon de conocimientos de muchas disciplinas sociales conduce a una situación paradojal. Así, el reconocimiento y la validación institucional suponen la pérdida o atenuación de la fuerza crítica de esos conocimientos respecto de esos mismos criterios de evaluación y enseñanza académica. En tal sentido, ciertos sectores del profesorado jurídico identificados con posicionamientos feministas son puestos bajo sospecha, por cuanto que no se eximen de ostentar prácticas consistentes con las lógicas de subalternación y exclusión universitaria, al tiempo que promueven conocimientos estandarizados y cercenados de su más radical sentido crítico.

Por fin, de los postulados feministas citados más arriba, es decir, de la crítica a la neutralidad del derecho, la asunción del conocimiento como praxis y la inevitable heterogeneidad del discurso jurídico, es posible inferir la crítica a la pretendida neutralidad de cualquier disciplina social y de todo enfoque epistemológico. En tal sentido, la neutralidad del género erigido desde una perspectiva general se revela cuanto menos problemático. En efecto, la idea de una perspectiva de género supone la congregación de múltiples posicionamientos en una sola mirada o comprensión unívoca respecto de esa categoría y todo lo que ella implica (Viturro, 2005). Por lo demás, si desde los feminismos se explicita el androcentrismo velado del derecho, cabe asimismo sospechar respecto de los privilegios y las jerarquías de quienes sustentan una perspectiva (parcial, singular, interesada) y las concesiones para su reconocimiento y legitimidad. La particularidad de la perspectiva de género, así como de toda perspectiva general, en la educación legal puede advertirse en los debates que demarcan el virtual campo de los feminismos jurídicos y de los estudios de género y de la sexualidad en el derecho. La 
suspicacia respecto de las paradojas de su institucionalización y el potencial detrimento en la radicalidad crítica es uno de los tantos puntos problemáticos que se plantean en la transversalización de los contenidos de género. Si la piedra de toque de las críticas feministas supuso señalar, como lo hiciera Olympe de Gouges, la falsa universalidad del sujeto del derecho, es necesario considerar cómo se sostiene, a costa de qué negaciones y obliteraciones, el género en términos de una perspectiva. Como afirma Paula Viturro (2005), "si los universalismos de la humanidad nos resultan sospechosos, demos también asumir que los universales del género también lo son" (pág. 300).

En suma, si se acepta que el derecho no es neutral, se admite necesariamente que la perspectiva de género tampoco lo es. La incorporación de temas y enfoques de los estudios de género y las sexualidades y feministas en la formación jurídica comprende entonces la apasionante tarea de debatir los modos en que esos contenidos inciden en la institución académica: en los mecanismos de validación y circulación del conocimiento, en las formas de la enseñanza y las jerarquías universitarias. Solo manteniendo la condición crítica y abierta de esos debates es posible desarticular las complicidades del Derecho con el androcentrismo y las desigualdades de género.

\section{Referencias bibliográficas}

Bartlett, Katharine T. \& Kennedy, Rosanne (edit.) (1991). Feminist Legal Theory. Westview Press: Oxford.
Bergallo, Paola \& Moreno, Aluminé (2017a). Antecedentes y Contextos. En Hacia políticas judiciales de género (pág. 41-66). Buenos Aires: Jusbaires Editorial.

(2017b). Hacia políticas judiciales de género. Buenos Aires: Jusbaires Editorial.

Costa, Malena (2015). El pensamiento jurídico feminista en los confines del Siglo xx. Asparkía. Investigación Feminista 26: (pág. 35-49).

Didot. (2016). Feminismos jurídicos. Buenos Aires: Ediciones

(2017). Feminismos jurídicos en Argentina. En Paola Bergallo \& Aluminé Moreno. (coord.). Hacia políticas judiciales de género (pág. 237-260). Buenos Aires: Jusbaires Editorial.

(2019) Feminismos jurídicos en la academia legal argentina. En Diana Maffía \& Aluminé Moreno (coord.). Miradas feministas sobre los derechos (pág. 33-48). Buenos Aires: Jusbaires Editorial.

Costa, Malena \& Lerussi, Romina (2018). Los feminismos jurídicos en la Argentina. Notas para pensar un campo emergente. Estudios Feministas, 26(1), pág. 1-13.

Kohen, Beatriz (2000). El feminismo jurídico en los países anglosajones: el debate actual. En Birgin, Haydeé (ed.), El derecho en el género y el género en el derecho (pág. 73-105). Buenos Aires: Biblos.

Maffía, Diana \& Moreno, Aluminé (coord.) (2019). Miradas feministas sobre los derechos. Buenos Aires: Jusbaires Editorial.

Olsen, Frances (ed.) (1995). Feminist Legal Theory (Vol II). Dartmouth: Aldershot.

(2000). El sexo del derecho. En Alicia Ruiz (edit.), Identidad femenina y discurso jurídico (pág. 25-44). Buenos Aires: Editorial Biblos.

Rodríguez, Ana Laura (2008). Las políticas sensibles al género: variedades conceptuales y desafíos de intervención, Temas y debates (pág. 109-129).

Rodríguez Manzano, Irene (2008). Mujeres y Naciones Unidas. Madrid: Catarata.

Viturro, Paula (2005). Constancias, Academia, Revista sobre enseñanza del derecho de Buenos Aires, pág. 295-300.

Weisberg, Kelly (edit.) (1993). Feminist Legal Theory. Foundations. Philadelphia: Temple University Press. 